\title{
Modeling of scalar mixing in turbulent jet flames by multiple mapping conditioning
}

\author{
K. Vogiatzaki, ${ }^{1}$ M. J. Cleary, ${ }^{2}$ A. Kronenburg, ${ }^{1, a)}$ and J. H. Kent ${ }^{3}$ \\ ${ }^{1}$ Department of Mechanical Engineering, Imperial College London, SW7 2AZ, United Kingdom \\ ${ }^{2}$ Division of Mechanical Engineering, The University of Queensland, Queensland 4072, Australia \\ ${ }^{3}$ School of Aerospace, Mechanical and Mechatronic Engineering, The University of Sydney, NSW 2006, \\ Australia
}

(Received 14 October 2008; accepted 22 December 2008; published online 25 February 2009)

\begin{abstract}
Multiple mapping conditioning (MMC) combines the probability density function (PDF) and the conditional moment closure (CMC) methods via the application of a generalized mapping function to a prescribed reference space. Stochastic and deterministic formulations of MMC exist, and the deterministic implementation has been applied here to a piloted jet diffusion flame (Sandia Flame D). This paper focuses on the feasibility of MMC and its closures for real (laboratory) flames and a relatively simple one-dimensional reference space that represents mixture fraction has been used. The remaining chemically reactive species are implicitly conditioned on mixture fraction and their fluctuations around the conditional mean are neglected. This work primarily evaluates the ability of the deterministic form of MMC to provide accurate and consistent closures for the mixture fraction PDF and the conditional scalar dissipation which do not rely on presumed shape functions for the PDF such as the commonly used $\beta$-PDF. Computed probability distributions agree well with measurements, and a detailed comparison of the modeled conditional and mean scalar dissipation with experimental data and conventional closures demonstrate MMC's potential. Predictions of reactive species and temperature are in good agreement with experimental data and similar in quality to singly conditioned, first-order CMC predictions. MMC therefore provides an attractive-since consistent-alternative approach for the modeling of scalar mixing in turbulent reacting flows. (C) 2009 American Institute of Physics. [DOI: 10.1063/1.3081553]
\end{abstract}

\section{INTRODUCTION}

The accurate prediction of turbulent small-scale scalar mixing is one of the key challenges of turbulence modeling due to its importance in, for example, heat transfer, atmospheric pollutant dispersion, and combustion. Scalar mixing is particularly relevant for reacting flows where fuel and oxidizer are issued separately into the combustion chamber and where the combustion process is largely controlled by turbulent mixing rather than chemical kinetics. The difficulty for the modeller is not only that turbulence is a stochastic phenomenon but also that scalar mixing is a molecular process which occurs at the smallest scales. The smallest scales are explicitly calculated in direct numerical simulations (DNSs) but computational expense limits application to flows with relatively low Reynolds numbers in simple geometries. Computational modeling of reacting flows of engineering interest is currently limited to Reynolds averaged Navier-Stokes approaches (RANS) and large-eddy simulations (LES). In both methods the smallest scales are not resolved and modeling is required to describe the scalar mixing.

In nonpremixed combustion the degree of scalar mixing is often characterized by the mixture fraction, $Z$, and in tur-

\footnotetext{
${ }^{a}$ Author to whom correspondence should be addressed. Present address: Institut für Technische Verbrennung, Universität Stuttgart, 70569 Stuttgart, Germany. Telephone: 49-711-68565635. Fax: 49-711-68555635. Electronic mail: kronenburg@itv.uni-stuttgart.de.
}

bulent flows this is a stochastic variable with a certain probability density function (PDF), $P_{Z}$. The PDF can be modeled with knowledge of its first and second moments and by assuming a certain shape such as a Gaussian or $\beta$-function. Normally, model equations are solved for the mean, $\tilde{Z}$, and variance, $\widetilde{Z^{\prime 2}}$, of mixture fraction. The rate of dissipation of mixture fraction variance is determined by the quantity $N_{Z}=D \nabla Z \nabla Z$ (called the scalar dissipation rate) where $D$ is the molecular diffusion coefficient. The importance of scalar dissipation rate to turbulent nonpremixed combustion is well known. Gradients of reactive species and temperature are correlated with gradients of the mixture fraction and therefore $N_{Z}$ controls the diffusional fluxes of these quantities to and from the reaction zone. The strong physical correlation between chemical source and scalar space diffusion terms is manifested in mixture fraction models such as the flamelet $^{1}$ and conditional moment closure $(\mathrm{CMC})$ methods $^{2-4}$ where the conditionally averaged dissipation $\left\langle N_{Z} \mid \eta\right\rangle$ $\equiv\langle D \nabla Z \nabla Z \mid \eta\rangle$ appears as an explicit parameter in the model formulation. Here $\eta$ is the sample space of mixture fraction. Its importance is not just limited to flamelet and CMC methods but extends to joint PDF combustion models ${ }^{5}$ where scalar dissipation conditioned on all scalars (not just on mixture fraction) appears as an unclosed parameter.

The unconditional Favre averaged scalar dissipation rate is related to the scalar variance by a dissipation time scale $\tau_{N}$ according to 


$$
\tilde{N}_{Z}=\frac{\widetilde{Z^{\prime 2}}}{\tau_{N}}
$$

It is common to assume that $\tau_{N}$ is proportional to the flow turbulence time scale, $\tau=\widetilde{k} / \widetilde{\varepsilon}$. Simple models which link $\left\langle N_{Z} \mid \eta\right\rangle$ to $\tilde{N}_{Z}$ have been developed but most are inconsistent with the PDF transport equation. ${ }^{4}$ Recent CMC based studies $^{4,6-9}$ derive the conditional scalar dissipation from the double integration of the mixture fraction PDF transport equation, thus enforcing consistency between $\left\langle N_{Z} \mid \eta\right\rangle$ and $P_{Z}$. Unfortunately this approach suffers from numerical problems in regions of low probability as the final model formula contains $P_{Z}$ in the denominator.

A class of conditional scalar dissipation models, applicable to CMC and joint PDF methods, are based on the concept of mapping closures. ${ }^{10-13}$ Mapping closures employ turbulent fluctuations and small-scale mixing in a mathematical reference space, whose PDF is known (or prescribed), to model the turbulent fluctuations and small-scale mixing in the physical composition space, whose PDF is not known in advance. In the past 5 years a new mapping closure model called multiple mapping conditioning (MMC) has been developed. ${ }^{14} \mathrm{MMC}$ shares the basic mapping closure ideas of the amplitude mapping closure (AMC) method of Chen et $a{ }^{10}{ }^{10}$ and includes all the major improvements suggested by Pope ${ }^{11}$ and Girimaji. ${ }^{12}$ However, MMC has the great advantage of being a generalized model applicable to all flow conditions. The earlier mapping closure methods derived expressions for the conditional scalar dissipation in composition space in terms of the conditional scalar dissipation in reference space. Unfortunately, the evaluation of the conditional scalar dissipation in reference space still required knowledge of the mean scalar gradients (i.e., two-point correlations) which could only be determined easily for Gaussian reference PDFs. ${ }^{10,11}$ Girimaji $^{12}$ extended the method by deriving a reference PDF transport equation and demonstrated that a $\beta$-PDF reference variable can also be used. In practice, however, the reference field PDF transport equation does not have a known solution and, strictly, Girimaji's method is applicable to homogeneous turbulence only. Apart from MMC, all the other models mentioned are external methods for calculating the conditional scalar dissipation. In contrast, MMC is a self-contained turbulent combustion model which solves the transport equations for turbulent scalar mixing and reaction in the reference space and conditional scalar dissipation is not explicitly present. The solved quantities in the MMC equations are called mapping functions which map between the reference space and the composition space. These equations were derived for generalized inhomogeneous turbulence and do not depend on the shape of the reference PDF. Furthermore, the composition joint PDF need not be presumed as it can be determined directly from the solved mapping functions.

The principal idea in MMC is the division of all turbulent fluctuations (and scalars) into major and minor groups. Turbulent fluctuations of minor scalars are correlated with the unrestricted fluctuations of the major scalars. A reference variable is assigned to each major scalar and the number of major scalars therefore determines the dimensionality of the model. MMC does not restrict the number of major scalars although in most flame cases of interest it should be possible to describe the composition space with a small number of well chosen primary variables ${ }^{14}$ and thus keeps computational costs low. The simplest form of MMC has only a single reference variable emulating the mixture fraction. Both probabilistic (or stochastic) and conditional (or deterministic) MMC model formulations are possible. In the conditional MMC, used here, fluctuations of minor scalars relative to major scalars are neglected and conditional reaction rates are modeled with first-order moments.

Although MMC is a generalized model, most applications to date have been for homogeneous, isotropic, decaying turbulence. Wandel and Klimenko ${ }^{15}$ used a stochastic approach where a single reference variable is mapped to mixture fraction. Cleary and co-worker ${ }^{16,17}$ applied a conditional MMC, where mixture fraction and normalized sensible enthalpy are chosen as major species. More recently, Cleary and Klimenko ${ }^{18}$ extended MMC modeling to inhomogeneous flows using a stochastic approach in the LES context. In the current work conditional MMC with a single reference variable for the mixture fraction is applied to an inhomogeneous reactive flow (Sandia Flame D).

It has been established previously that many combustion models accurately predict reactive species in Flame D and therefore the emphasis in the current work is to investigate the suitability of MMC as a generalized scalar mixing model. The conditional MMC model is presented in generalized form in Sec. II. In Sec. III the test case configuration, the specific MMC model formulation conditioned on the mixture fraction, and closure considerations are discussed in detail. The accuracy of the MMC mixing field statistics (mean, standard deviation and PDF) are compared to experimental data and conventional RANS mixing field predictions in Sec. IV and detailed analysis of the conditional scalar dissipation, which can be extracted from the MMC solution, is given in Sec. V. In Sec. VI we determine the sensitivity of reactive scalars to different scalar mixing models and conclusions are made in Sec. VII.

\section{THE MMC MODEL}

A detailed derivation of the MMC model and its conventional closure assumptions are given in Klimenko and Pope. ${ }^{14}$ Here, we summarize the general equations that will form the basis for further simplifications introduced in Sec. III below. The MMC equation for the spatial and temporal evolution of the mapping function, $X_{I}=X_{I}(\boldsymbol{\xi} ; \boldsymbol{x}, t)$, is given by

$$
\frac{\partial X_{I}}{\partial t}+\boldsymbol{U} \nabla X_{I}+A_{k} \frac{\partial X_{I}}{\partial \xi_{k}}-B_{k l} \frac{\partial^{2} X_{I}}{\partial \xi_{k} \xi_{l}}=W_{I},
$$

where the reference space is $\boldsymbol{\xi}=\left(\xi_{1}, \xi_{2}, \ldots, \xi_{\text {major }}\right)$, upper case subscript $I$ denotes all scalars (major and minor) and lower case subscripts $k$ and $l$ denote the major scalars only.

In Eq. (2) $\boldsymbol{U}, A_{k}$, and $B_{k l}$ are the coefficients of velocity, drift, and diffusion, respectively, whose closures are obtained through consistency with the following joint reference PDF transport equation: ${ }^{14}$ 


$$
\frac{\partial \bar{\rho} P_{\xi}}{\partial t}+\nabla \boldsymbol{U} \bar{\rho} P_{\xi}+\frac{\partial A_{k} \bar{\rho} P_{\xi}}{\partial \xi_{k}}+\frac{\partial^{2} B_{k l} \bar{\rho} P_{\xi}}{\partial \xi_{k} \xi_{l}}=0
$$

For a jointly Gaussian PDF with zero mean and unity variance, Eq. (3) is satisfied if the MMC velocity and drift coefficients follow the relationships ${ }^{14}$

$$
\begin{aligned}
& \boldsymbol{U}=\boldsymbol{U}(\xi ; x, t)=\boldsymbol{U}^{(0)}+\boldsymbol{U}_{k}^{(1)} \xi_{k}, \\
& A_{k}=-\frac{\partial B_{k l}}{\partial \xi_{l}}+B_{k l} \xi_{l}+\frac{1}{\bar{\rho}} \nabla \bar{\rho} \boldsymbol{U}_{k}^{(1)}, \\
& \boldsymbol{U}^{(0)}=\widetilde{\mathbf{u}}, \\
& \boldsymbol{U}_{k}^{(1)}\left\langle\xi_{k} X_{i}\right\rangle^{*}=\widetilde{\mathbf{u}^{\prime} Y_{i}^{\prime}},
\end{aligned}
$$

where $\mathbf{u}$ is the velocity vector and $Y_{i}$ is the scalar composition modeled by mapping function (i.e. $Y_{i}=\left\langle X_{i}\right\rangle^{*}$ ). The notation used for different averages is the following: terms with an overtilde are Favre averages of turbulent flow field quantities; terms in simple angular brackets represent Favre averages evaluated with the use of the PDF for mixture fraction, $P_{Z}$; and the star is added to the angular brackets to denote Favre averages evaluated with the use of the reference space PDF, $P_{\xi}$. Note that the reference PDF does not have to be Gaussian, and the coefficients can be determined for any reasonable choice of the reference PDF. However, the modeling of $\boldsymbol{U}$ and $A_{k}$ will involve much more complex dependencies than those given in Eqs. (4) and (5) to satisfy Eq. (3) and a simple implementation may then not be feasible.

Equations (4)-(7) imply that $B_{k l}$ can be treated as an independent coefficient (this will be discussed in detail in Sec. III). Following convention, ${ }^{14} B_{k l}$ is modeled independently of $\boldsymbol{\xi}\left(B_{k l}=B_{k l}(\boldsymbol{x}, t)\right)$ and is related to the Favre averaged scalar dissipation tensor $\widetilde{N}_{i j}$ by

$$
B_{k l}\left\langle\frac{\partial X_{i}}{\partial \xi_{k}} \frac{\partial X_{j}}{\partial \xi_{l}}\right\rangle^{*}=\tilde{N}_{i j} .
$$

It is apparent from Eq. (8) that closure of the MMC model requires knowledge of the unconditional scalar dissipation of the major scalars, but it does not explicitly include the more difficult to model conditional scalar dissipation. The components of the tensor $\widetilde{N}_{i j}$ may be modeled with scaling relations similar to Eq. (1). In Sec. V we show how the conditional scalar dissipation can be extracted in postprocess analysis from the mapping function gradients in $\boldsymbol{\xi}$-space.

\section{THE MODEL IMPLEMENTATION}

In the previous section the MMC model was presented as a generalized model without nominating the major scalars. The fact any choice and any number of major scalars is possible while maintaining the same equation form is one of the advantages of the method. For the present case the mixture fraction is selected as the only major scalar and the specific model implementation and the associated numerical scheme are discussed below. Example calculations illustrate the importance of some closure details and therefore the experimental test case is described at this stage.

\section{A. Case configuration}

The MMC implementation is validated against experimental multiscalar measurements in a piloted methane-air jet flame (Sandia Flame D). ${ }^{19}$ The fuel composition for Flame D is $25 \%$ methane and $75 \%$ air by volume. The burner geometry features an axisymmetric fuel jet with diameter of $D=7.2 \mathrm{~mm}$ and a surrounding pilot with outer diameter of $18.2 \mathrm{~mm}$. The exit velocity of the jet is $49.6 \mathrm{~m} / \mathrm{s}$ and of the pilot is $11.4 \mathrm{~m} / \mathrm{s}$.

Sandia Flame D is well characterized experimentally through extensive scalar measurements. A large number of modeling attempts have been performed in the context of both RANS and LES using different combustion approaches. ${ }^{6,20-22}$ Therefore the primary focus of the current paper is to investigate the suitability of MMC model as a generalized scalar mixing model.

\section{B. Mixture fraction evolution}

The mixture fraction is chosen as the only major scalar and the multidimensional reference space is replaced by a single reference variable $\xi=\xi_{Z}$. Eq. (2) holds for the major and minor scalars but in this section we concentrate on the mapping function for mixture fraction which we denote by $X_{Z}$. The evolution of $X_{Z}$ is given by

$$
\frac{\partial X_{Z}}{\partial t}+\boldsymbol{U} \nabla X_{Z}+A \frac{\partial X_{Z}}{\partial \xi}-B \frac{\partial^{2} X_{Z}}{\partial \xi^{2}}=0
$$

For clarity the subscript $Z$ has been dropped from the drift and diffusion coefficients which are understood to operate in $\xi$-space. The numerical implementation employs the spatially conservative form of Eq. (9) given by

$$
\nabla \bar{\rho} \boldsymbol{U} X_{Z}+\bar{\rho} A \frac{\partial X_{Z}}{\partial \xi}-\bar{\rho} B \frac{\partial^{2} X_{Z}}{\partial \xi^{2}}=X_{Z} \xi \nabla \bar{\rho} \boldsymbol{U}^{(1)},
$$

where $\bar{\rho}$ is the Reynolds averaged density and time derivatives have been neglected due to the steady nature of the flow under investigation. Eq. (10) is discretized with a finite volume scheme and coupled to an axisymmetric, elliptic RANS-CFD code called GENPOL. ${ }^{9}$ Flow turbulence is modeled in accordance with the $k-\varepsilon$ model of Jones and Launder ${ }^{23}$ with the standard constants: $C_{\varepsilon 1}=1.44, C_{\varepsilon 2}=1.92$, $\sigma_{k}=1.0, \sigma_{\varepsilon}=1.3$, and $C_{\mu}=0.09$.

The mapping function $X_{Z}$ maps between $\xi$, whose statistical details are fully prescribed, and $Z$. Therefore knowledge of $X_{Z}$ implies knowledge of all statistical properties of $Z$. For example, the first and second moments are given by

$$
\left\langle X_{Z}\right\rangle^{*}=\int_{-\infty}^{\infty} X_{Z} P_{\xi} d \xi
$$

and

$$
\left\langle X_{Z}^{\prime 2}\right\rangle^{*}=\int_{-\infty}^{\infty}\left(X_{Z}-\left\langle X_{Z}\right\rangle^{*}\right)^{2} P_{\xi} d \xi
$$




\section{MMC model closures}

Models for the conditional velocity, drift, and diffusion coefficients are required to close Eq. (10) and the requirement for consistency with Eq. (3) poses restrictions on that modeling. In the present study we model all coefficients according to Eqs. (4)-(7) as proposed by Klimenko and Pope. ${ }^{14}$ Although the suggested coefficients are consistent with Eq. (3) this does not imply that they are unique. Taking Eq. (3) for a spatially and temporally invariant Gaussian reference PDF as a starting point and expanding the partial derivatives we obtain

$$
\nabla \boldsymbol{U} \bar{\rho} P_{\xi}+\frac{\partial A \bar{\rho} P_{\xi}}{\partial \xi}+\frac{\partial}{\partial \xi}\left(\bar{\rho} P_{\xi} \frac{\partial B}{\partial \xi}-\xi B \bar{\rho} P_{\xi}\right)=0
$$

and integration over $\xi$-space gives

$$
A=\left(-\frac{\partial B}{\partial \xi}+B \xi\right)-\frac{1}{\bar{\rho} P_{\xi}} \int \nabla \boldsymbol{U} \bar{\rho} P_{\xi} d \xi .
$$

Based only on the mathematical constraints of the problem it is apparent that coefficients $\boldsymbol{U}$ and $B$ can be any reasonable functions of $\boldsymbol{x}$ and $\xi$. We then determine $A$ from Eq. (14) to ensure that the reference PDF transport equation is satisfied. Eq. (14) demonstrates that the range of choices of a model for $A$ is broader than suggested in Eq. (5) and depends on the choice of $\boldsymbol{U}$ and $B$. The modeling of these two terms is discussed in Secs. III C 1 and III C 2.

\section{Conditional velocity}

A linear model given by Eq. (4) is used for the conditional velocity. It has a similar form to the linear model for velocity conditioned on the mixture fraction which is commonly used in CMC computations. ${ }^{4}$ The gradient of $\boldsymbol{U}$ in $\xi$-space, denoted $\boldsymbol{U}^{(1)}$, is given by Eq. (7). Following convention, we model the Favre turbulent flux $\widetilde{\mathbf{u}^{\prime} Z^{\prime}}$ according to the gradient diffusion hypothesis so that

$$
\widetilde{\mathbf{u}^{\prime} Z^{\prime}}=-\frac{\mu_{t}}{\sigma_{z}} \nabla\left\langle X_{Z}\right\rangle^{*}
$$

The turbulent diffusivity is modeled as $\mu_{t}=C_{\mu} \bar{\rho} \widetilde{k}^{2} / \widetilde{\varepsilon}$ and the turbulent Schmidt number $\sigma_{z}$ is set to 0.7 .

Since it quantifies the effect of turbulence on the evolution of $X_{Z}, \boldsymbol{U}^{(1)}$ is one of the most important terms in the MMC model. Its role is explored in Fig. 1 where profiles of $X_{Z}$ over reference space are presented at different axial locations and at $r / D=1$ which is in the vicinity of the shear layer. Three alternative test cases are compared to the reference case where $\boldsymbol{U}^{(1)}$ is as described above. In case $1, \boldsymbol{U}^{(1)}$ is omitted from the calculation of the conditional velocity in Eq. (4) and from the drift term in Eq. (5). As expected for this case turbulent fluctuations are not generated by the model and the $X_{Z}$ profile is horizontal; representing a delta PDF in mixture fraction space. In case $2, \boldsymbol{U}^{(1)}$ is omitted from the conditional velocity model in Eq. (4) only. As for case $1, X_{Z}$ is horizontal in $\xi$-space at all locations and this result indicates that it is through conditional velocity, specifically, that turbulent fluctuations are generated. In case $3, \boldsymbol{U}^{(1)}$ is omitted from the calculation of the drift coefficient in Eq.
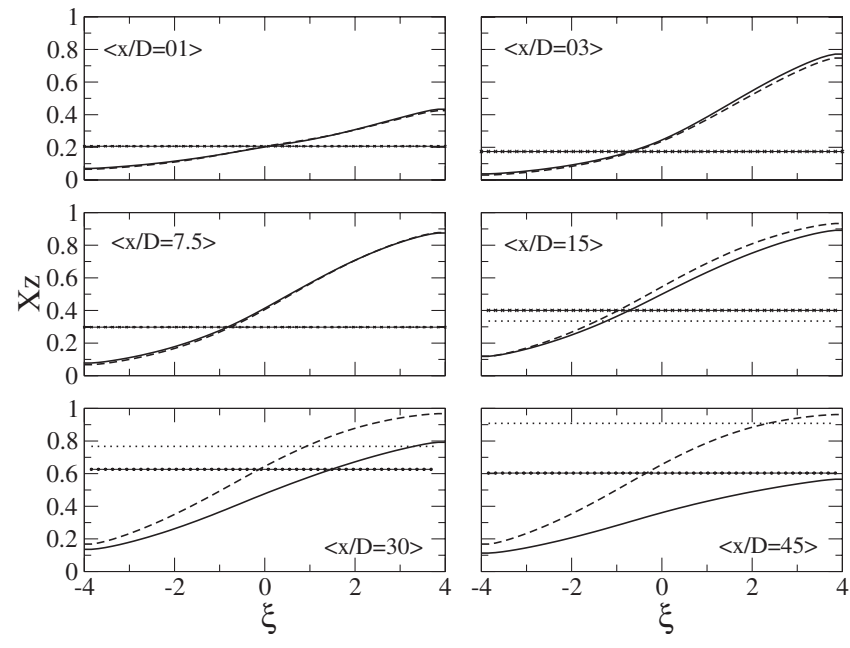

FIG. 1. Profiles of the mixture fraction mapping function, $X_{Z}$, in reference space at various axial locations and $r / D=1$. Solid lines are the reference case, dotted lines: case 1, stars: case 2, and dashed lines: case 3.

(5) but retained in the velocity model in Eq. (4). This results in a stronger dependence of $X_{Z}$ on $\xi$ with increasing downstream location, implying the physically unrealistic situation of the mixture fraction variance increasing in the far field.

The analysis above illustrates that while $\boldsymbol{U}^{(1)}$ generates turbulent fluctuations through the conditional velocity, the gradient term containing $\boldsymbol{U}^{(1)}$ in the drift coefficient acts to dissipate those fluctuations. The correct balancing of these two opposing forces is necessary to accurately predict jet breakup and flame length. Although from a mathematical view point many different models for $\boldsymbol{U}$ may be permitted this does not imply that all such models are good. The suitability of the MMC model with the nominated linear conditional velocity model is assessed against experimental data and the conventional RANS solutions for the scalar mixing field in Sec. IV, but further work is required to investigate the existence of alternate, superior models for $\boldsymbol{U}$.

Depending on the turbulence conditions, the modeling of $\boldsymbol{U}^{(1)}$ may not be well defined. Some minimum level of fluctuations needs to be imposed to avoid $\left\langle\xi X_{Z}\right\rangle^{*} \rightarrow 0$ leading to $\boldsymbol{U}^{(1)} \rightarrow \infty$. Here we set the minimum value $\left\langle\xi X_{Z}\right\rangle^{*}$ as $10^{-8}$ to avoid any subsequent numerical problems.

\section{Diffusion coefficient}

In MMC it is conventional to model the diffusion coefficients, $B_{k l}$, independently of the reference variables. Therefore according to Eq. (8) the diffusion coefficient in $\xi$-space must satisfy the relation

$$
B\left\langle\frac{\partial X_{Z}}{\partial \xi} \frac{\partial X_{Z}}{\partial \xi}\right\rangle^{*}=\tilde{N}_{Z}
$$

An external model is required for $\tilde{N}_{Z}$ but this should not be considered a restrictive factor as mapping closures can accommodate any model for mean scalar dissipation. For MMC specifically, $\tilde{N}_{Z}$ and the other input parameter $\widetilde{\mathbf{u}}$, connect the transport equation in the artificial mathematical reference space with the physical turbulent flow field, thus giving the mapping functions a physical meaning. In the present 
work $\tilde{N}_{Z}$ in Eq. (16) is obtained from the turbulent mixing parameters $\widetilde{k}$ and $\widetilde{\varepsilon}$ and the scalar variance as

$$
\tilde{N}_{Z}=C_{Z} \frac{\widetilde{\varepsilon}}{\tilde{k}}\left\langle X_{Z}^{\prime 2}\right\rangle^{*}
$$

where the constant $C_{Z}$ is set to unity.

\section{Numerical scheme}

Equation (10) is discretized on an axisymmetric finite volume grid which is staggered so that velocity is determined at the cell boundaries and $X_{Z}$ at the cell centers. The computational domain extends $80 \mathrm{~cm}$ vertically and $5 \mathrm{~cm}$ radially and is discretized by $175 \times 60$ cells in the axial and radial directions, respectively. It is refined near the fuel port and pilot. For the reference variable $\xi, 50$ cells cover the interval $\xi \in[-4,4]$. The term on the right hand side of Eq. (10) is modeled with the diffusion approximation of Eq. (15). It is normal practice to move such terms to the left hand side of the equation for implicit inclusion in the finite volume convection scheme (e.g., see Ref. 9). However here the right hand side is multiplied by the product $X_{Z} \xi$ so we instead treat the term explicitly with a second-order approximation for the second derivative. In the absence of a balancing spatial diffusion flux on the left hand side of Eq. (10) a numerically stable first-order upwinding scheme is used for advective transport. For fluxes in reference space, a hybrid scheme is used which changes from second-order central differencing to first-order upwind differencing when the magnitude of the Peclet number exceeds two. Boundary conditions are defined in both physical and reference spaces. In the $\xi$-plane the two boundary cells are set dynamically so that $\partial^{2} X_{Z} / \partial \xi^{2}=0$. The solver for $X_{Z}$ uses the biconjugated gradient method producing results for all spatial and $\xi$-space locations simultaneously. Values for $\boldsymbol{U}, A$, and $B$ are computed explicitly, based on the solution at the previous iteration.

\section{E. Conventional RANS scalar mixing model}

In RANS modeling it is conventional to model the first two moments of the scalar mixing field directly. Here we denote the conventional RANS first and second mixture fraction moments as $\tilde{f}$ and $\widetilde{f^{\prime 2}}$, respectively. For turbulent flow in axisymmetric coordinates these quantities can be modeled as

$$
\bar{\rho} \tilde{u} \frac{\partial \tilde{f}}{\partial x}+\bar{\rho} \tilde{v} \frac{\partial \tilde{f}}{\partial r}=\frac{\partial}{\partial x}\left(\frac{\mu_{t}}{\sigma_{f}} \frac{\partial \tilde{f}}{\partial x}\right)+\frac{1}{r} \frac{\partial}{\partial r}\left(r \frac{\mu_{t}}{\sigma_{f}} \frac{\partial \tilde{f}}{\partial r}\right)
$$

and

$$
\begin{aligned}
\bar{\rho} \widetilde{u} \frac{\partial f^{\prime 2}}{\partial x}+\bar{\rho} \widetilde{v} \frac{\partial f^{\prime 2}}{\partial r}= & 2 \frac{\mu_{t}}{\sigma_{g}}\left(\frac{\partial f^{\prime 2}}{\partial x}\right)^{2}+2 \frac{\mu_{t}}{\sigma_{g}}\left(\frac{\partial f^{\prime 2}}{\partial r}\right)^{2} \\
& +\frac{\partial}{\partial x}\left(\frac{\mu_{t}}{\sigma_{g}} \frac{\widetilde{f^{\prime 2}}}{\partial x}\right)+\frac{1}{r} \frac{\partial}{\partial r}\left(r \frac{\mu_{t}}{\sigma_{g}} \frac{\widetilde{f^{\prime 2}}}{\partial r}\right) \\
& -2 \widetilde{\rho} \widetilde{N}_{Z} .
\end{aligned}
$$

Here, $\tilde{u}$ and $\tilde{v}$ are the Favre averaged axial and radial flow velocities and the turbulent Schmidt numbers $\sigma_{f}$ and $\sigma_{g}$ are

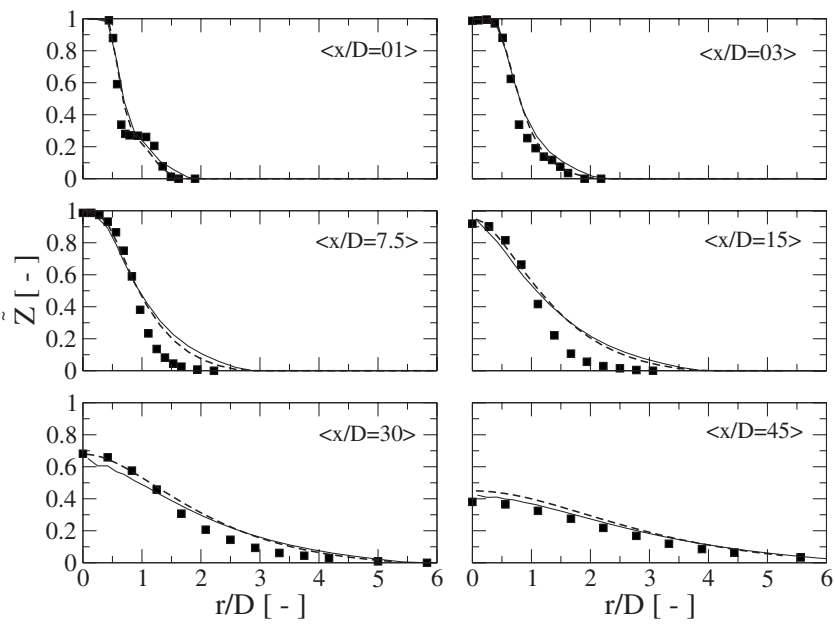

FIG. 2. Radial profiles of mean mixture fraction. Squares are experimental data (Ref. 19), solid lines are MMC predictions, and dashed lines are conventional RANS predictions given by the solution of Eq. (18).

set to 0.7. Similar to MMC, the mean scalar dissipation in Eq. (19) is given by

$$
\tilde{N}_{Z}=C_{Z} \frac{\widetilde{\varepsilon}}{\widetilde{k}} \widetilde{f}^{\prime 2}
$$

Provided the model equations for $X_{Z}, \tilde{f}$, and $\widetilde{f^{\prime 2}}$ are accurate and implemented correctly then $\left\langle X_{Z}\right\rangle^{*}$ and $\tilde{f}$ are two alternative models for $\tilde{Z}$, and $\left\langle X_{Z}^{\prime 2}\right\rangle^{*}$ and $\widetilde{f^{\prime 2}}$ are two alternative models for $\widetilde{Z^{\prime 2}}$. The flow turbulence determines the turbulent scalar mixing through the models for $\mu_{t}$ and $\tilde{N}_{Z}$ which are identical in both the MMC and conventional RANS mixing formulations above. The linear closure of the conditional velocity given by Eq. (4) is the only additional modeling contained in MMC that does not appear in conventional RANS. Therefore any differences between the predicted scalar mixing fields will be related to the modeling of this term.

The advantage of MMC is that all the statistics of $Z$ and not just the mean and variance are contained in solution for $X_{Z}$. In this work Eqs. (18) and (19) are used primarily to provide a comparison for the MMC predictions. For numerical convenience the $\tilde{f}$ and $\widetilde{f^{\prime 2}}$ fields are developed initially with Eqs. (18) and (19) and used for setting the $X_{Z}$ initial conditions.

\section{MIXING FIELD STATISTICS}

Figures 2 and 3 show radial profiles of $\left\langle X_{Z}\right\rangle^{*}$ and $\left\langle X_{Z, \mathrm{rms}}\right\rangle^{*}=\sqrt{\left\langle X_{Z}^{\prime 2}\right\rangle^{*}}$ at several axial locations. Overall the results are in good agreement with the experimental data and qualitatively very similar to other published computations of this flame (see, e.g., Refs. 20-22). The mean mixture fraction is well predicted along the centerline at all axial locations shown, however it is noticeably overpredicted in the shear layer at $x / D=7.5$ and $x / D=15$. The rms is also well predicted along the centerline but peak values in the shear layer are somewhat overpredicted close to the nozzle and underpredicted for $x / D>15$. Near the nozzle the model is unable to fully capture the double-peaked rms but further down- 

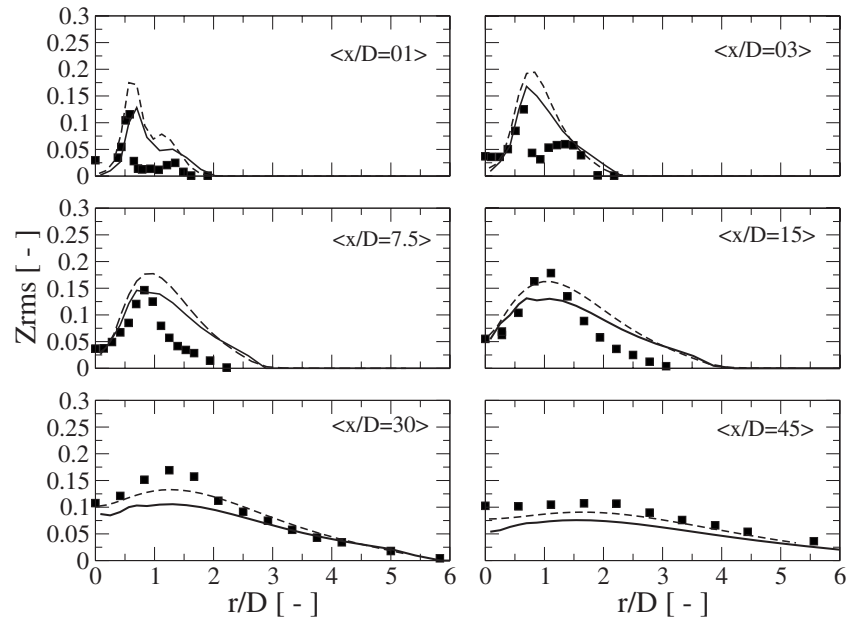

FIG. 3. Radial profiles of rms of mixture fraction. Squares are experimental data (Ref. 19), solid lines are MMC predictions, and dashed lines are conventional RANS predictions given by the solution of Eq. (19).

stream where a single radial peak exists the predicted trends are correct. The accuracy of the MMC predictions is put into context by comparison with the $\tilde{f}$ and $\tilde{f}_{\text {rms }}=\sqrt{\tilde{f}^{\prime 2}}$ computations using Eqs. (18) and (19) which are also included in Figs. 2 and 3. The similarity of the predicted mixing field statistics from MMC and conventional RANS is not surprising. The key closures that control the production and dissipation of scalar fluctuations are identical for both methods. The mean dissipation is modeled by Eqs. (17) and (20), and the modeling of the turbulent scalar flux is based on the gradient diffusion hypothesis in both cases. It is therefore rather surprising that small but distinct differences in the modeling of the mixture fraction rms field persist. An analysis of the modeling of the turbulent flux of the scalar variance and the role of the linear conditional velocity helps explain these differences and this is outlined in the Appendix.

In addition to the mean and variance, the mixture fraction PDF can be recovered from the solution for $X_{Z}$ :

$$
P_{Z}=P_{\xi}\left(\frac{d X_{Z}}{d \xi}\right)^{-1} \text {. }
$$

Figure 4 shows MMC predicted $P_{Z}$ at various axial and radial locations alongside experimental data, ${ }^{24}$ and $\beta$-function PDFs computed with the MMC mean and variance given by Eqs. (11) and (12), respectively. Flamelet and CMC combustion models commonly presume a $\beta$-function PDF for mixture fraction and the very good agreement between $P_{Z}$ from MMC and the corresponding $\beta$-function was therefore expected. We reiterate that MMC does not need to presume $P_{Z}$ and, in fact, the MMC framework given by Eq. (2) can be applied unaltered to cases where the PDF is not a $\beta$-function. MMC predictions of a bimodal PDF for a reaction progress variable in homogeneous turbulence are reported elsewhere ${ }^{17}$ and an extension to laboratory flames is in progress. Compared to the experimental data the PDF shapes and the locations of maximum $P_{Z}$ in $Z$-space are very well reproduced near the nozzle, but further downstream the peak values of
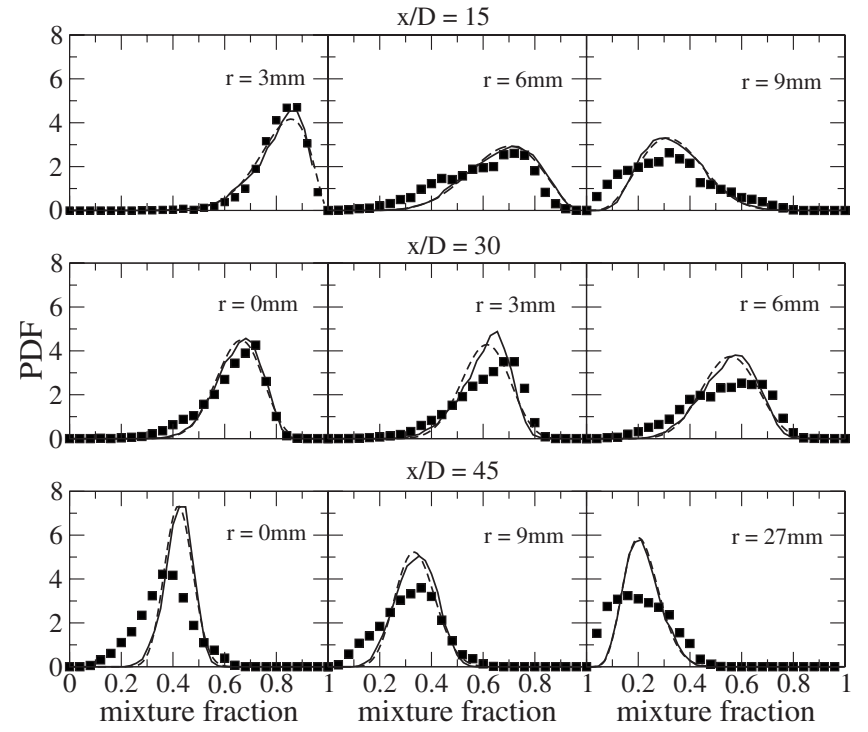

FIG. 4. Mixture fraction PDF at various locations. Squares are experimental data (Ref. 24), solid lines are MMC predictions, and dashed lines are $\beta$-PDFs with mean and variance given by Eqs. (11) and (12).

$P_{Z}$ are generally overpredicted. These outcomes are consistent with the MMC predictions for mean and rms. Also MMC fails to predict the experimentally observed level PDF skewness at $x / D=45$ and other far downstream locations. More complex expressions for the MMC closures, in particular relaxation of the assumptions of linearity for $\boldsymbol{U}(\xi)$ [see Eq. (4)] may be necessary to overcome this deficiency. However any such change in the velocity closure will remain speculative without detailed analysis of an adequate DNS database and, therefore, it is not attempted here. It suffices to say that MMC in its current implementation provides the conditional statistics that are needed for combustion modeling and this is discussed in Secs. VI and VII.

\section{CONDITIONAL SCALAR DISSIPATION}

\section{A. The MMC model for conditional scalar dissipation}

The scalar dissipation conditioned on the mixture fraction does not appear explicitly in the MMC equations, however Klimenko showed in a conference contribution that it can be determined from the mapping function solutions for $X_{Z}$ through a transformation from the reference space, $\xi$, to the mixture fraction sample space, $\eta$. This procedure is described in detail by Cleary and Kronenburg. ${ }^{16}$ In the context of the present work the transformed nonconservative form of the MMC equation for minor scalars is

$$
\frac{\partial Q_{\alpha}}{\partial t}+\hat{\boldsymbol{U}} \nabla Q_{\alpha}-\hat{B} \frac{\partial^{2} Q_{\alpha}}{\partial \eta^{2}}=\hat{W}_{\alpha}
$$

The quantities in Eq. (22) are

$$
\begin{aligned}
& Q_{\alpha}=\left\langle X_{\alpha} \mid \eta\right\rangle, \\
& \hat{\boldsymbol{U}}=\boldsymbol{U},
\end{aligned}
$$



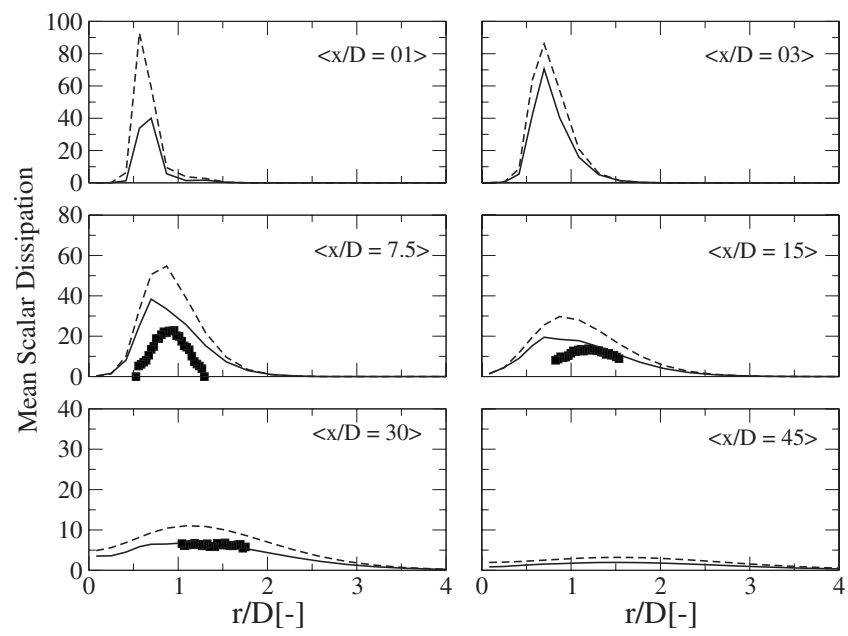

FIG. 5. Radial profiles of mean scalar dissipation at various axial locations. Squares are experimental data (Refs. 25 and 26), solid lines are MMC predictions, and dashed lines are conventional RANS predictions using Eqs. (19) and (20).

$$
\hat{B}=B\left(\frac{\partial X_{Z}}{\partial \xi}\right)^{2}
$$

and

$$
\hat{W}_{\alpha}=\left\langle W_{\alpha} \mid \eta\right\rangle \text {. }
$$

Equation (22) is equivalent to the singly conditioned, firstorder CMC equation for high Reynolds number flows. Of particular importance to the modeling of scalar mixing is that $\hat{B}$ appears in place of, and is therefore a model for, the conditional scalar dissipation

$$
\left\langle N_{Z} \mid \eta\right\rangle \simeq B\left(\frac{\partial X_{Z}}{\partial \xi}\right)^{2}
$$

\section{B. Model validation}

The MMC model for $\left\langle N_{Z} \mid \eta\right\rangle$ given by Eq. (27) is validated against experimental data and two other $\left\langle N_{Z} \mid \eta\right\rangle$ models which are commonly used in CMC computations: the amplitude mapping closure (AMC) model $^{10}$ (sometimes called the inverse error function model); and the doubly integrated PDF transport equation model. ${ }^{8}$ The alternative models were briefly discussed in Sec. I and a detailed explanation of their performance for the flame conditions considered is given by Sreedhara et $a l^{6}$

The three conditional scalar dissipation models depend directly (MMC and AMC) or indirectly (PDF integration model) on $\tilde{N}_{Z}$. For MMC, $\tilde{N}_{Z}$ is given by Eq. (17) and for the alternative methods, which rely on the conventional RANS scalar mixing model, $\tilde{N}_{Z}$ is given by Eq. (20). Figure 5 displays radial profiles of predicted $\tilde{N}_{Z}$ at six axial locations alongside one-dimensional (1D) radial line-imaging experimental data ${ }^{25,26}$ which are available at three axial locations only. Quantitatively both MMC and conventional RANS overpredict $\tilde{N}_{Z}$, particularly in the region close to the nozzle. This is consistent with the modeled rms of mixture fraction

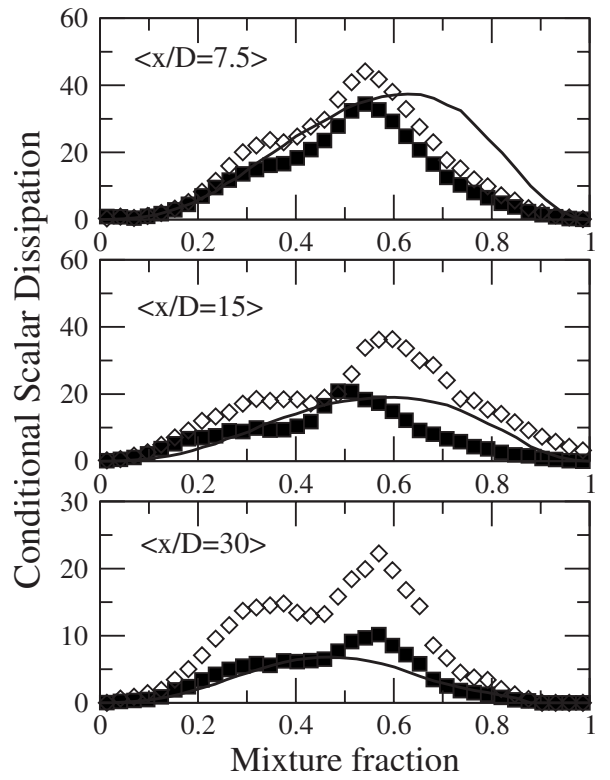

FIG. 6. Profiles of radially averaged conditional scalar dissipation in mixture fraction space. Squares and diamonds are 1D and 3D experimental data, respectively (Ref. 24), and solid lines are the MMC model.

being greater than experimental $\mathrm{rms}$ in that region of the flow. Qualitatively the predicted $\tilde{N}_{Z}$ trends are in agreement with the experimental data; the radial locations of the peak values are predicted quite well for $x / D=7.5$ and $x / D=30$ but some minor discrepancies occur at $x / D=15$.

Conditional scalar dissipation by MMC is compared to $1 \mathrm{D}$ radial line-imaging and a limited set of three-dimensional (3D) point measurements in Fig. 6. The data are weighted by the PDF and averaged in the radial direction according to

$$
\left\langle N_{Z} \mid \eta, x\right\rangle_{R}=\frac{\int\left\langle N_{Z}(x, r) \mid \eta\right\rangle P_{Z}(\eta, x, r) 2 \pi r d r}{\int P_{Z}(\eta, x, r) 2 \pi r d r} .
$$

Near the fuel jet scalar gradients in the radial direction are dominant and hence 1D and 3D experimental data are similar. Further downstream where the flow becomes isotropic gradients in the axial and circumferential direction are also important and the 3D results are quantitatively more accurate. MMC predicted $\left\langle N_{Z} \mid \eta, x\right\rangle_{R}$ is in good qualitative and quantitative agreement with the measurements at $x / D=7.5$. However peak values are underpredicted at greater axial locations, most notably at $x / D=30$, and this is a direct consequence of the underpredicted variance as shown in Fig. 3. In addition MMC does not capture the double-peak profile evident in the 3D data which has a second peak near $\eta=0.6$.

Further qualitative comparison is now made with the 1D radial line-imaging experimental data. Figure 7 shows $\left\langle N_{Z} \mid \eta\right\rangle$ profiles in mixture fraction space at various axial and radial locations. AMC and doubly integrated PDF model predictions are also shown. A deficiency of the AMC model is that $\left\langle N_{Z} \mid \eta\right\rangle$ must peak at $\eta=0.5$ whereas the alternative models permit an asymmetric profile. It is observed that the MMC reproduces the profile shapes and the location of the peak better than the doubly integrated PDF method. The MMC also predicts the radial dependence of $\left\langle N_{Z} \mid \eta\right\rangle$ more satisfactorily than the other methods. 


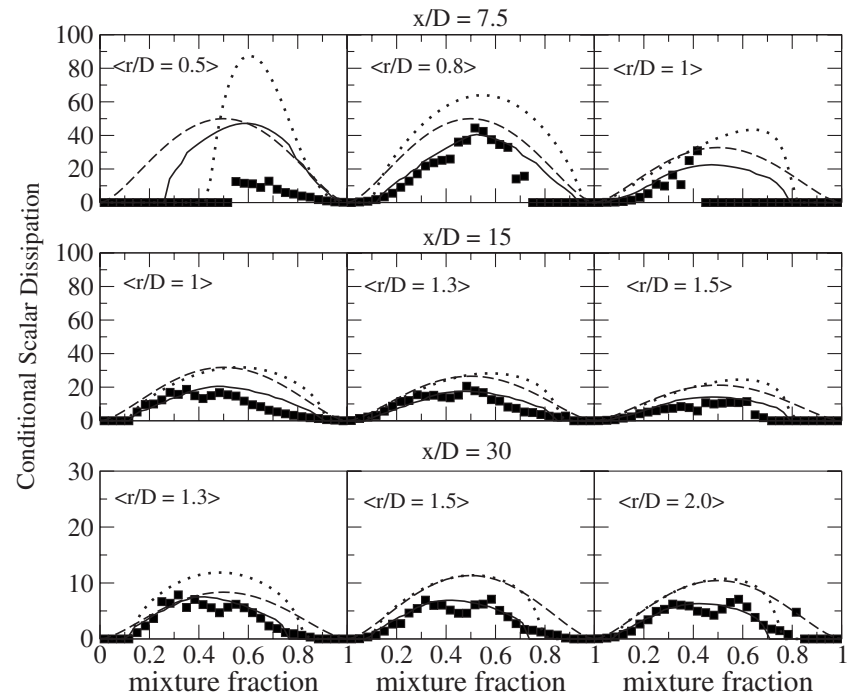

FIG. 7. Profiles of local conditional scalar dissipation in mixture fraction space. Squares are 1D experimental data (Ref. 24), solid lines are the MMC model, dotted lines are the doubly integrated PDF model, and dashed lines are the AMC model.

\section{REACTIVE SCALAR MODELING}

The most obvious implementation of MMC for modeling nonpremixed reacting flows is via Eq. (10) with the replacement of $X_{Z}$, the mapping function for mixture fraction, by $X_{I}$, the mapping function for all scalars. However, the transformation in Eq. (22) to the CMC form of the MMC model facilitates an analysis of the sensitivity of species predictions to the modeling of conditional scalar dissipation. Therefore the CMC form has been used for the present study and conditional scalar dissipation is modeled by MMC, AMC and the doubly integrated PDF model. The CMC combustion model and the numerical scheme used for this purpose have been described in detail previously. ${ }^{9}$ Species predictions are compared to the experimental data for Sandia Flame D. ${ }^{19,24}$ The modeling uses a 35 species and 219-step reaction mechanism (GRI3.0) and the effects of radiation are included.

Figures 8-10 present radial profiles of unconditional temperature and unconditional mass fraction of $\mathrm{CO}$ and $\mathrm{OH}$. For MMC the PDF for convoluting the unconditional averages is given by Eq. (21) while for AMC and the PDF integration method a presumed $\beta$-function is used with mean and variance given by $\tilde{f}$ and $\widetilde{f^{\prime 2}}$, respectively. Generally, temperature and species predictions for all three model cases are in good agreement with the experimental data and each other. However, there is a discrepancy with the experimental data at $x / D=7.5$ and $x / D=15$. Examination of the conditional average temperature, $\mathrm{CO}$ and $\mathrm{OH}$ profiles presented in Fig. 11 reveal that in mixture fraction space predictions are in excellent agreement with experiments at $x / D=15$ and, although not shown, similarly good results are found at $x / D=7.5$. Therefore the inaccuracy of the unconditional predictions is directly attributable to the overprediction of mean mixture fraction at these axial locations for $r / D>1$ (see Fig. 2). The predicted mixture fraction, by both MMC and conventional RANS, is in the vicinity of the stoichiometric

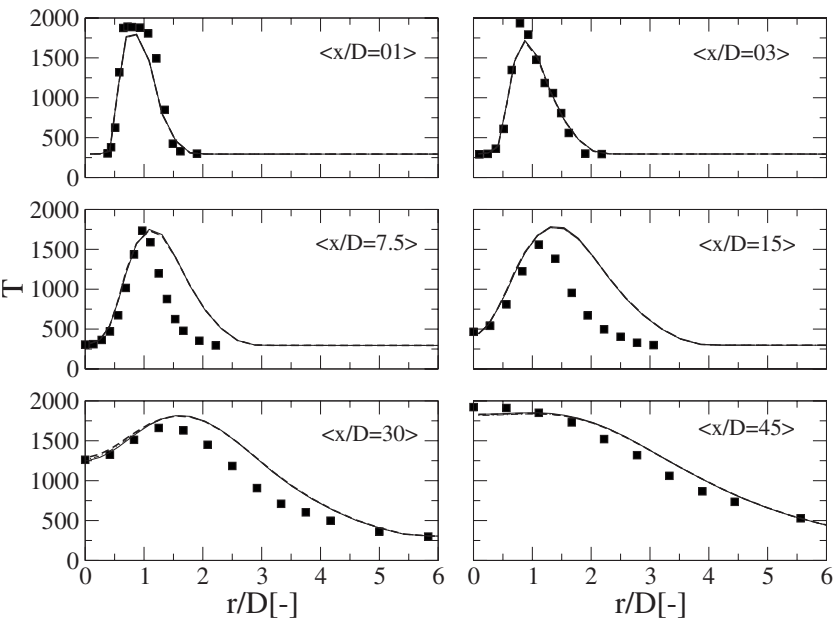

FIG. 8. Radial profiles of mean temperature. Squares are experimental data (Ref. 19), solid lines are predictions using the MMC closure for conditional scalar dissipation, dashed lines are predictions using the AMC closure, and dotted lines are predictions using the doubly integrated PDF closure.

value over a greater radial distance than is observed in experiments and hence the predicted temperature and species are closer to their stoichiometric conditional means beyond $r / D=1$.

Very little difference is observed between the predictions of conditional (Fig. 11) and unconditional (Fig. 8) temperature for the three different model cases. The same can be said for $\mathrm{CH}_{4}, \mathrm{O}_{2}, \mathrm{CO}_{2}$, and $\mathrm{H}_{2} \mathrm{O}$. This outcome is expected for the principal reactive scalars which are not greatly affected by the relatively small $\left\langle N_{Z} \mid \eta\right\rangle$ differences between the three test cases. However, some small differences between the model predictions for conditional $\mathrm{CO}$ and $\mathrm{OH}$ are evident in Fig. 11 with the MMC giving slightly more accurate results. In general all three models perform quite well although MMC produces the most accurate results for peak $\mathrm{OH}$ and for rich side $\mathrm{CO}$ at $x / D>30$. These modest improvements are attributed to the improved predictions for the conditional scalar dissipation.
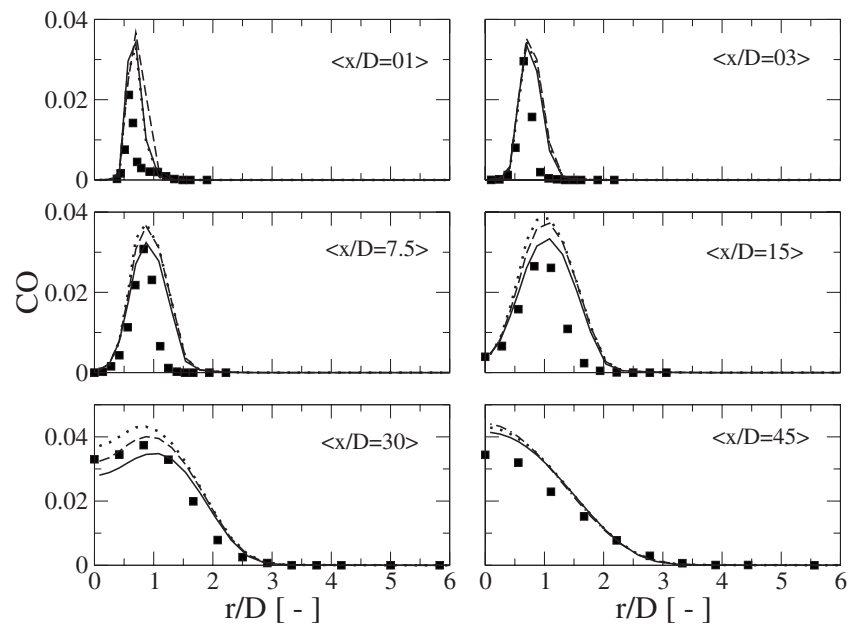

FIG. 9. Radial profiles of mean CO. Squares are experimental data (Ref. 19), solid lines are predictions using the MMC closure for conditional scalar dissipation, dashed lines are predictions using the AMC closure, and dotted lines are predictions using the doubly integrated PDF closure. 

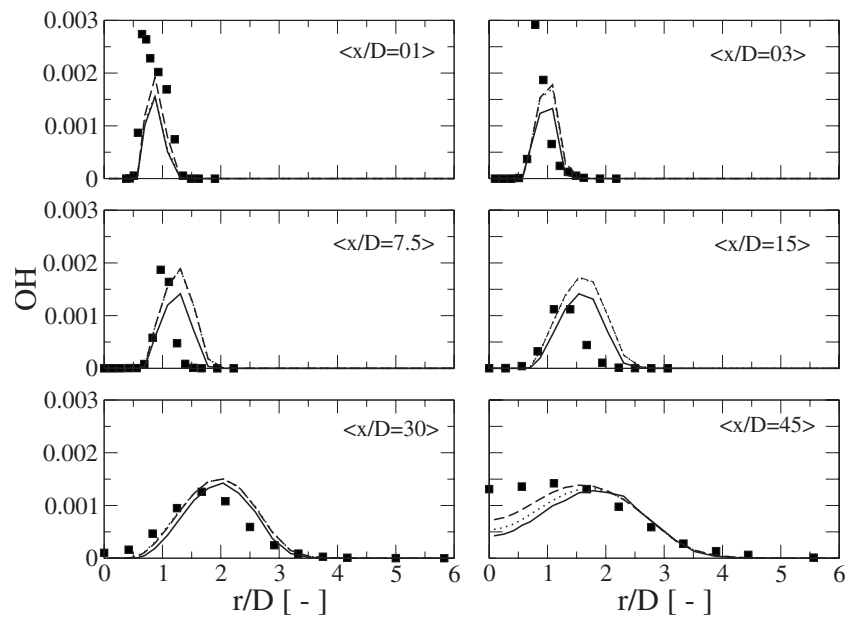

FIG. 10. Radial profiles of mean OH. Squares are experimental data (Ref. 19), solid lines are predictions using the MMC closure for conditional scalar dissipation, dashed lines are predictions using the AMC closure, and dotted lines are predictions using the doubly integrated PDF closure.

The inaccuracy of the unconditional reactive scalar predictions at some locations (most notably at $x / D=7.5$ and $x / D=15)$ should not be interpreted as a weakness in the generalized MMC framework. The inability to fully capture the rate of spread and breakdown of the fuel jet is a characteristic of the $k-\varepsilon$ turbulence model and similar results for the mixing field are reported by others (e.g., Ref. 27). Flame D computations with turbulence closures based on the
Reynolds stress model ${ }^{28}$ (RSM) and LES (Ref. 21) have produced very accurate mixture fraction field results. The present MMC formulation could be introduced almost unchanged into RSM and LES based CFD codes. Based on the very good agreement between predicted and experimental conditional averages in Fig. 11 it is reasonable to expect that if MMC was coupled with a superior turbulence model the predictions of unconditional reactive scalars would also be better than those shown in Figs. 8-10. As demonstrated here MMC produces only slightly improved conditional average species results relative to models with conditional scalar dissipation closures based on AMC and the doubly integrated PDF model. However, the considerable advantages of MMC relative to those alternatives, especially if implemented in the form of Eq. (10), are that the conditional scalar dissipation appears in closed form, the mixture fraction PDF does not need to be presumed, and those two quantities are automatically consistent with each other.

\section{CONCLUSION}

This paper has investigated the suitability of MMC as a generalized scalar mixing model for turbulent reacting flows. In MMC the scalar space is divided into major and minor groups. The turbulent fluctuations of minor scalars are correlated with the unrestricted fluctuations of the major scalars, each of which is assigned a reference variable with a known or prescribed PDF. In the present work the conditional form of MMC was used which restricts the minor scalars so that
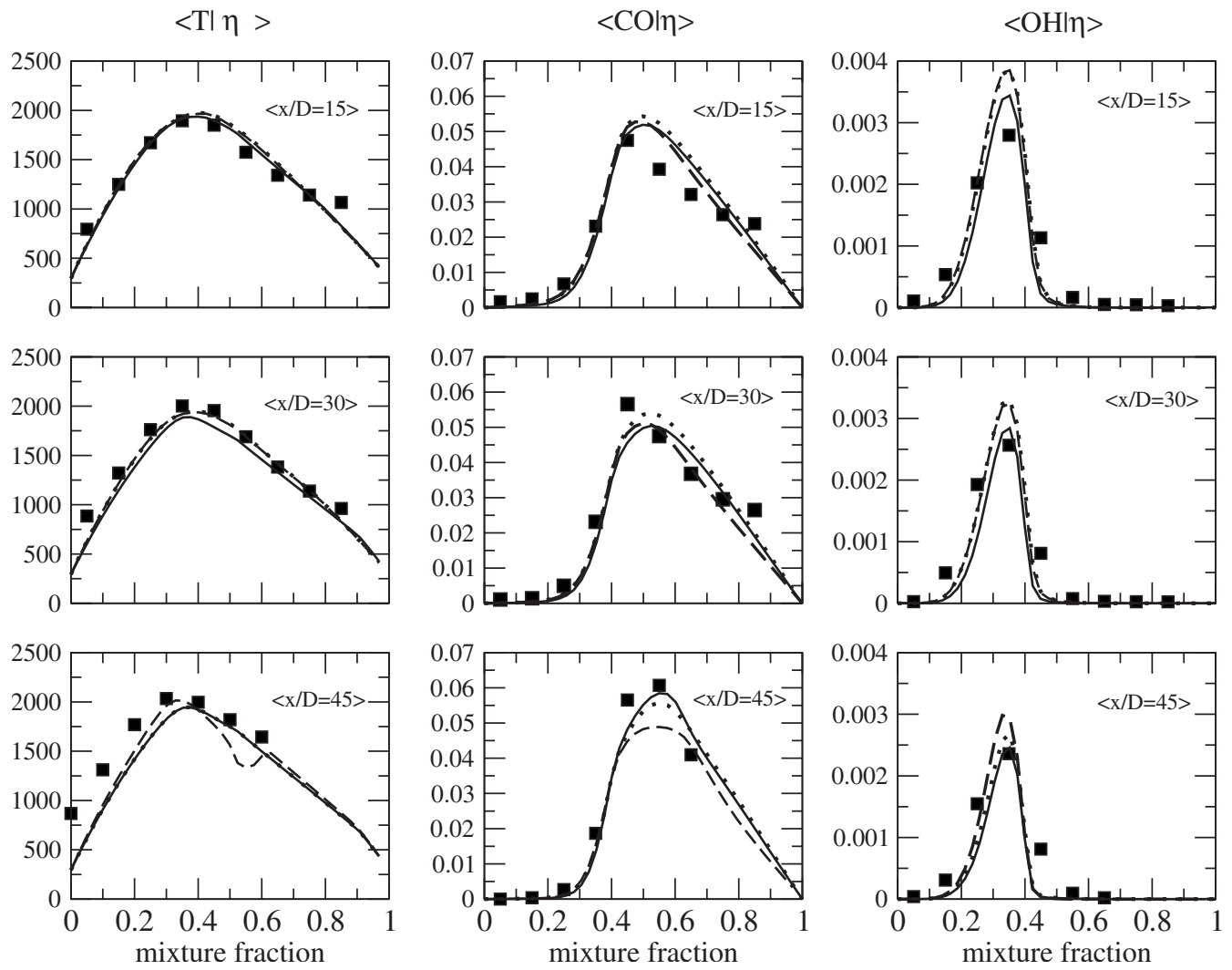

FIG. 11. Profiles of conditionally averaged temperature, $\mathrm{CO}$ and $\mathrm{OH}$ in mixture fraction space. Squares are experimental data (Ref. 19), solid lines are predictions using the MMC closure for conditional scalar dissipation, dashed lines are predictions using the AMC closure, and dotted lines are predictions using the doubly integrated PDF closure. 
they can fluctuate only jointly with the major scalars. For the nonpremixed flame conditions considered turbulent scalar statistics are suitably parametrized by the mixture fraction and this was selected as the only major scalar. The model solves transport equations for mapping functions which map between the reference and physical scalar spaces. It was shown that from the solved mapping function for the mixture fraction and the prescribed reference PDF one can determine the mixture fraction PDF and, of course, all its moments. The great advantage of MMC is that the difficult to model conditional scalar dissipation is contained implicitly and there is no need for external closure models.

The performance of MMC as a generalized scalar mixing model was tested against experimental data in a nonpremixed jet flame (Sandia Flame D). Trends for the first two moments of mixture fraction were well reproduced, although local underprediction of the rms is evident at some far field locations. MMC results were also compared to the solution of conventional RANS equations for the scalar mean and variance. Predictions by the two methods were generally very similar and, as the turbulence model was the same, the small differences can be attributed to the MMC conditional velocity which was modeled linearly in reference space. The MMC predicted mixture fraction PDF closely resembles a $\beta$-function and agreement with experimental data was quite good although due to the underprediction of scalar variance the peak values of the predicted PDF were too high in the far field.

Although the conditional scalar dissipation does not appear in the MMC model equations it can be determined through a coordinate transformation between the reference space which has a Gaussian PDF and the mixture fraction sample space. This casts MMC in the same form as singly conditioned, first-order CMC but with three significant advantages: the conditional scalar dissipation appears in closed form; the mixture fraction PDF is known and does not need to be presumed; and those two quantities are automatically consistent with each other. Detailed comparisons of the MMC closure for the conditional scalar dissipation rate with experimental data, and the AMC and doubly integrated PDF transport equation models show that MMC is a qualitatively better model. It was shown that the quantitative accuracy depends on the quality of the model for mean scalar dissipation upon which MMC makes not restrictions. The sensitivity of reactive species predictions to the conditional scalar dissipation was also tested. As expected for a flame with low levels of local extinction the principal reactive scalars were shown to be relatively insensitive, however, MMC predictions of intermediate and incomplete combustion products improved modestly relative to the alternative models.

Since the current work is one of the first implementations of MMC only one major scalar was used in order to demonstrate the feasibility of the method. However the MMC approach is not limited to a single, nonreacting major scalar and implementation for multiple and reactive major scalars is part of ongoing work to model flames with significant local quenching. Furthermore it is noted that the approach could be easily included in a large-eddy simulation.

\section{ACKNOWLEDGEMENTS}

The authors acknowledge the financial support by the EPSRC under Grant No. GR/T22766 and we would like to thank Dr. R.S. Barlow for providing the scalar dissipation data.

\section{APPENDIX: CONSISTENCY BETWEEN MMC AND THE SCALAR VARIANCE EQUATION}

MMC is PDF compliant if the general form of the parameters $\boldsymbol{U}, A_{k}$, and $B_{k l}$ are chosen so that Eq. (3) is satisfied. However, the accuracy of MMC depends on the specific closures chosen, in particular, on the modeling for the velocity coefficient. This parameter is analyzed in detail below.

$\boldsymbol{U}(\xi)$ is a model for the conditional velocity $\langle\boldsymbol{u} \mid \eta\rangle$ that can be decomposed into the unconditional mean and a fluctuation around this mean that depends on $\eta,\langle\boldsymbol{u} \mid \eta\rangle=\tilde{\boldsymbol{u}}+\mathbf{u}_{\eta}^{\prime}$. The starting point for the analysis is the steady state mixture fraction PDF transport equation

$$
\nabla\left[\bar{\rho}\left(\tilde{\boldsymbol{u}}+\mathbf{u}_{\eta}^{\prime}\right) P_{Z}\right]=-\frac{\partial^{2}}{\partial \eta^{2}}\left(\bar{\rho}\left\langle N_{Z} \mid \eta\right\rangle P_{Z}\right) .
$$

The above equation can be multiplied by mixture fraction and integration over the mixture fraction space yields

$$
\nabla(\bar{\rho} \tilde{\boldsymbol{u}} \widetilde{Z})+\nabla\left(\bar{\rho} \int_{0}^{1} \eta \boldsymbol{u}_{\eta}^{\prime} P_{Z} d \eta\right)=0,
$$

which is equivalent to the high Reynolds number governing equation for $\tilde{Z}$,

$$
\nabla(\bar{\rho} \tilde{\boldsymbol{u}} \tilde{Z})+\nabla\left(\widetilde{\rho} \widetilde{\boldsymbol{u}^{\prime} Z^{\prime}}\right)=0
$$

Equating Eqs. (A2) and (A3) provides a sufficient condition for the integral in Eq. (A2),

$$
\int_{0}^{1} \eta u_{\eta}^{\prime} P_{Z} d \eta=\widetilde{u^{\prime} Z^{\prime}}
$$

Replacing $\eta$ in the above with $X_{Z}(\xi)$ and upon using the linear model for the MMC conditional velocity [Eq. (4)] we get

$$
\begin{aligned}
\int_{0}^{1} \eta \boldsymbol{u}_{\eta}^{\prime} P_{Z} d \eta & =\int_{-\infty}^{\infty} X_{Z}(\xi) \boldsymbol{u}_{\eta}^{\prime} P_{\xi} d \xi \\
& =\int_{-\infty}^{\infty} X_{Z}(\xi) \boldsymbol{U}^{(1)} \xi P_{\xi} d \xi \\
& =\boldsymbol{U}^{(1)} \int_{-\infty}^{\infty} X_{Z}(\xi) \xi P_{\xi} d \xi=\boldsymbol{U}^{(1)}\left\langle X_{Z} \xi\right\rangle^{*}
\end{aligned}
$$

Modeling $\boldsymbol{U}^{(1)}$ according to Eq. (7) reproduces Eq. (A4) thus illustrating consistency with the first moment of the PDF transport equation.

We now apply a similar procedure for the second moment. Multiplication of the PDF transport equation by $Z^{2}$, integration in mixture fraction space and comparison with the mixture fraction variance equation gives 

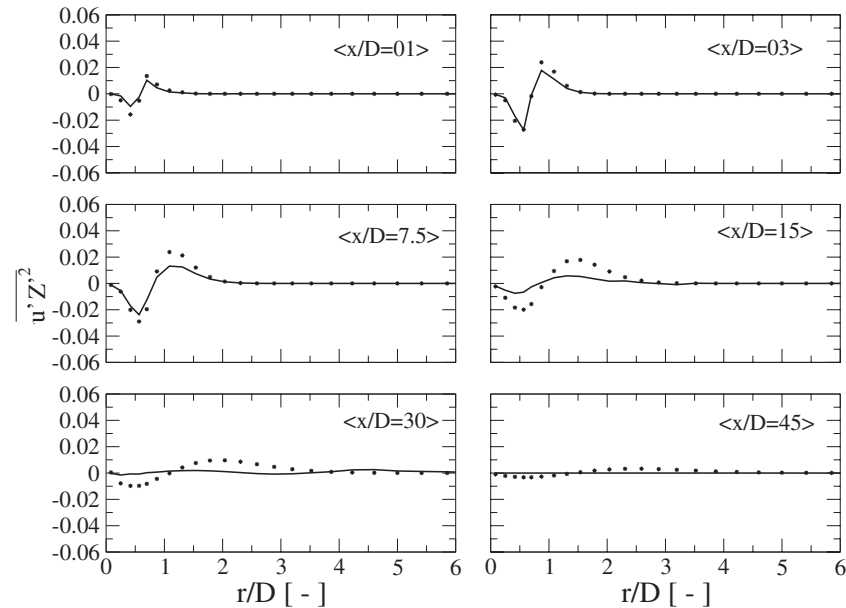

0.06

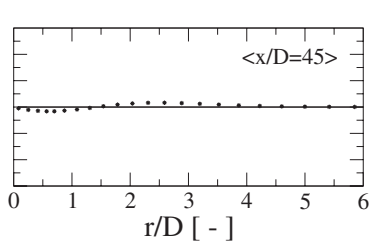

FIG. 12. Radial profiles of the turbulent flux of scalar variance, $\widetilde{\boldsymbol{u}^{\prime} \boldsymbol{Z}^{\prime 2}}$. The solid lines are results from MMC [left hand side of Eq. (A7)] and symbols represent modeling via a standard gradient diffusion approximation [first two terms on right hand side of Eq. (19)].

$$
\int_{0}^{1}\left(\eta^{2}-2 \tilde{Z} \eta\right) \boldsymbol{u}_{\eta}^{\prime} P_{Z} d \eta=\widetilde{\boldsymbol{u}^{\prime} Z^{\prime 2}}
$$

Replacing $\boldsymbol{u}_{\eta}^{\prime}$ in Eq. (A6) by $\boldsymbol{U}^{(1)} \xi$ leads to the relationship

$$
\frac{\widetilde{u^{\prime} Z^{\prime}}\left\langle X_{Z}^{\prime 2} \xi\right\rangle^{*}}{\left\langle X_{Z} \xi\right\rangle^{*}}=\widetilde{u^{\prime} Z^{\prime 2}}
$$

In MMC the left hand side of Eq. (A7) can be evaluated with the aid of Eq. (15) which models the turbulent flux of the scalar mean via gradient diffusion. In conventional RANS a separate variance equation is solved and the turbulent flux of the scalar variance is also modeled by a gradient method [see the first two terms on RHS of Eq. (19)]. This additional modeling requirement in the variance transport equation gives rise to differences in conventional RANS and MMC. Figure 12 compares the MMC and gradient diffusion models for $\widetilde{\boldsymbol{u}^{\prime} Z^{\prime 2}}$. Differences between the models are largest in the shear layer particularly at $x / D=15$ and $x / D=30$. The discrepancies between the MMC and conventional RANS predictions of the scalar variance shown in Fig. 3 can now be explained by these observed differences in its modeled turbulent flux.

\footnotetext{
${ }^{1}$ N. Peters, Turbulent Combustion (Cambridge University Press, Cambridge, 2000).

${ }^{2}$ A. Y. Klimenko, "Multicomponent diffusion of various admixtures in turbulent flow," Fluid Dyn. 25, 327 (1990).

${ }^{3}$ R. W. Bilger, "Conditional moment closure for turbulent reacting flows," Phys. Fluids A 5, 436 (1993).

${ }^{4}$ A. Y. Klimenko and R. W. Bilger, "Conditional moment closure for turbulent combustion," Prog. Energy Combust. Sci. 25, 595 (1999).
}

${ }^{5}$ S. B. Pope, "PDF methods for turbulent reactive flows," Prog. Energy Combust. Sci. 11, 119 (1985).

${ }^{6}$ S. Sreedhara, Y. Lee, K. Y. Huh, and D.H. Ahn, "Comparison of submodels for conditional velocity and scalar dissipation in CMC simulation of piloted jet and bluff-body flames," Combust. Flame 152, 282 (2008).

${ }^{7}$ A. Kronenburg, R. W. Bilger, and J. H. Kent, "Computation of conditional average scalar dissipation in turbulent jet diffusion flames," Flow, Turbul. Combust. 64, 145 (2000).

${ }^{8}$ C. B. Devaud, R. W. Bilger, and T. Liu, "A new method for modeling the conditional scalar dissipation rate," Phys. Fluids 16, 2004 (2004).

${ }^{9}$ M. J. Cleary and J. H. Kent, "Modelling of species in hood fires by conditional moment closure," Combust. Flame 143, 357 (2005).

${ }^{10} \mathrm{H}$. Chen, S. Chen, and R. H. Kraichnan, "Probability distribution of a stochastically advected scalar field," Phys. Rev. Lett. 63, 2657 (1989).

${ }^{11}$ S. B. Pope, "Mapping closures for turbulent mixing and reaction," Theor. Comput. Fluid Dyn. 2, 255 (1991).

${ }^{12} \mathrm{~S}$. S. Girimaji, "A mapping closure for turbulent scalar mixing using a time-evolving reference field," Phys. Fluids A 4, 2875 (1992).

${ }^{13} \mathrm{~F}$. Gao, "Mapping closure and non-Gaussianity of the scalar probability density functions in isotropic turbulence," Phys. Fluids A 3, 2438 (1991).

${ }^{14}$ A. Y. Klimenko and S. B. Pope, "The modelling of turbulent reactive flows based on multiple mapping conditioning," Phys. Fluids 15, 1907 (2003).

${ }^{15}$ A. P. Wandel and A. Y. Klimenko, "Testing multiple mapping conditioning mixing for Monte Carlo probability density function simulations," Phys. Fluids 17, 128105 (2005).

${ }^{16}$ M. J. Cleary and A. Kronenburg, "Hybrid multiple mapping conditioning on passive and reactive scalars," Combust. Flame 151, 623 (2007).

${ }^{17}$ A. Kronenburg and M. J. Cleary, "Multiple mapping conditioning for flames with partial premixing," Combust. Flame 155, 215 (2008).

${ }^{18}$ M. J. Cleary and A. Y. Klimenko, "A generalised multiple mapping conditioning approach for turbulent combustion," Flow, Turbul. Combust., http://www.springerlink.com/content/18813k700401831r/ (2008).

${ }^{19}$ R. S. Barlow and J. H. Frank, "Piloted $\mathrm{CH}_{4} /$ air flames C, D, E, and F-Release 2.0," Sandia National Laboratories CA, 2003.

${ }^{20} \mathrm{H}$. Pitsch, "Improved pollutant predictions in large-eddy simulations of turbulent non-premixed combustion by considering scalar dissipation rate fluctuations," Proceedings of the Combustion Institute, 2002, Vol. 29, pp. 1971-1978.

${ }^{21}$ S. Navarro-Martinez, A. Kronenburg, and F. Di Mare, "Conditional moment closure for large eddy simulations," Flow, Turbul. Combust. 75, 245 (2005).

${ }^{22}$ A. Kempf, A. F. Flemming, and J. Janicka, "Investigation of lengthscales, scalar dissipation, and flame orientation in a piloted diffusion flame by LES," Proceedings of the Combustion Institute, 2005, Vol. 30, pp. 557-565.

${ }^{23}$ R. P. Jones and B. E. Launder, "Calculation of low-Reynolds number phenomena with a 2-equation model of turbulence," Int. J. Heat Mass Transfer 15, 301 (1972).

${ }^{24}$ R. S. Barlow, J. H. Frank, A. N. Karpetis, and J. Y. Chen, "Piloted methane/air jet flames: Transport effects and aspects of scalar structure," Combust. Flame 143, 433 (2005).

${ }^{25}$ A. N. Karpetis and R. S. Barlow, "Measurements of flame orientation and scalar dissipation in turbulent partially premixed methane flames," Proceedings of the Combustion Institute, 2005, Vol. 30, pp. 665-672.

${ }^{26}$ R. S. Barlow and A. N. Karpetis, "Scalar length scales and spatial averaging effects in turbulent piloted methane/air jet flames," Proceedings of the Combustion Institute, 2005, Vol. 30, pp. 673-680.

${ }^{27}$ S. H. Kim and K. Y. Huh, "Second-order conditional moment closure modeling of turbulent piloted jet diffusion flames," Combust. Flame 138, 336 (2004).

${ }^{28}$ A. Habibi, B. Merci, and D. Roekaerts, "Turbulence radiation interaction in Reynolds-averaged Navier-Stokes simulations of nonpremixed piloted turbulent laboratory-scale flames," Combust. Flame 151, 303 (2007). 